\title{
Miguel Sánchez-Ostiz entre réalité et fiction
}

Jean-Pierre Castellani

\section{OpenEdition}

\section{Journals}

Édition électronique

URL : https://journals.openedition.org/cher/3834

DOI : $10.4000 /$ cher.3834

ISSN : 2803-5992

\section{Éditeur}

Presses universitaires de Strasbourg

\section{Édition imprimée}

Date de publication : 1 décembre 2015

Pagination : 185-196

ISBN : 978-2-86820-913-9

ISSN : 1968-035X

\section{Référence électronique}

Jean-Pierre Castellani, « Miguel Sánchez-Ostiz entre réalité et fiction », reCHERches [En ligne], 15 | 2015, mis en ligne le 01 décembre 2021, consulté le 14 décembre 2021. URL : http:// journals.openedition.org/cher/3834; DOI : https://doi.org/10.4000/cher.3834

Ce(tte) œuvre est mise à disposition selon les termes de la Licence Creative Commons Attribution Pas d'Utilisation Commerciale - Partage dans les Mêmes Conditions 4.0 International. 


\title{
Miguel Sánchez-Ostiz entre réalité et fiction
}

\author{
Jean-Pierre Castellani \\ Université François-Rabelais de Tours
}

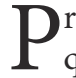
rofesseur des universités, Miguel Sánchez-Ostiz est un auteur très prolifique qui publie, depuis 1982, un nombre incalculable de textes aux genres souvent hybrides, entre fictions, autobiographies, mémoires, pamphlets, récits de voyages et journaux personnels, recueils poétiques, essais littéraires, éditions de catalogues de peintres. Écrivain qui se situe volontairement en dehors du politiquement correct et institutionnel, doté d'un esprit libertaire et adoptant une position indépendante face à la problématique basque, Sánchez-Ostiz n’est pas traduit en langue française et fait figure souvent, en Espagne, d'écrivain maudit et marginalisé, inclassable, incohérent et provocateur. Une partie de la critique estime même qu'il écrit trop, et trop vite, qu'il se répète et devient chaotique. Au point qu'il est parfois difficile d'attribuer un genre bien précis à ses textes et donc de les recevoir en toute clarté. Pourtant sa production offre un cas exemplaire de rénovation du genre romanesque qu'il est intéressant d'analyser.
\end{abstract}

Depuis ses premiers livres, en particulier Las pirañas (1992) jusqu'aux plus récents comme La flecha del miedo (2000), ou Cornejas de Bucarest (2010), Sánchez-Ostiz présente une mise en cause féroce de la réalité sociale et politique de l'Espagne depuis la dernière guerre carliste jusqu'à la démocratie actuelle, et plus précisément une dénonciation implacable de la société basque et de la ville de Pampelune, perçues comme un guignol burlesque.

Pourtant, nous pensons que ce serait une erreur de réduire le statut de Sánchez-Ostiz à celui d'un romancier soucieux uniquement de situer ses histoires dans le décor réel de l'histoire contemporaine. Nous estimons même qu'apparaît, chez lui, de plus en plus, une tendance à offrir des histoires où se mêlent des éléments réalistes et des caractéristiques propres au monde du rêve, ou plus exactement du cauchemar, avec une forte présence de l'imagination. Au point d'inventer ou de revendiquer un genre nouveau, dans ce qu'il appelle lui-même «la ensoñación autobiográfica» (Sánchez-Ostiz 2010: 270), genre nouveau et parfois déroutant qui en fait un des écrivains les plus attachants de sa génération. 
Le personnage dominant et prédominant des romans de Sánchez-Ostiz est un narrateur, avocat de profession, qui mène une enquête: par exemple, autour de la mort mystérieuse d'un de ses amis, le philosophe Miguel Arróniz, dans El corazón de la niebla (2001), la disparition d'une figure du carlisme du XIX e siècle, Tristán de Barraute dans En Bayona bajo los porches (2002), la possible existence d'un fils du peintre Gustavo de Maetzu dans La nave de Baco (2004), la personnalité extraordinaire de l'aventurier Telmo Gamecho à la poursuite des secrets du trésor de l'île de Juan Fernández dans La calavera de Robinson (2007), la découverte d'un cadavre dans une carrière de pierre dans Zarabanda (2011)1.

Sachant que la formation initiale de Sánchez-Ostiz est juridique, le lecteur peut d'abord penser qu'il s'agit d'une littérature de témoignage, enrichie d'une bonne dose de la personnalité de l'auteur. Mais il convient très vite de nuancer cette identification de l'auteur et de son narrateur dans la mesure où, dans $L a$ flecha del miedo (2000), le narrateur est un ventriloque, c'est-à-dire quelqu'un qui sévade de la réalité en exerçant cette activité artificielle qui consiste à prêter sa voix à trois marionnettes ${ }^{2}$.

Remarquons la portée métaphorique du titre: comme le suggère une épigraphe, cette flèche de la peur est tirée du Psaume 90 de la Bible qui nous parle d'un homme coupable, à cause de sa méchanceté, de ses mensonges et de sa lâcheté, et un Dieu qui le remet dans le bon chemin grâce à ses conseils, dont l'un dit: «No temerás el espanto nocturno/ni la flecha que vuela de día.» L'illustration de la couverture confirme cette situation avec un montage esthétique d'Alfonso Ponce de León qui met en scène un homme aliéné qui subit un accident et vit terrorisé par ses fantasmes.

Par ailleurs, le ventriloque nous fournit l'image d'un écrivain qui parle au nom des autres, et transmet, dissimulé derrière ses marionnettes, le message iconoclaste qui caractérise en général l'engagement de Sánchez-Ostiz dans tous ses écrits ${ }^{3}$.

1 Dans ce roman, ce sont six voix de proches du protagoniste qui racontent les crimes passés, composant ainsi un chœur confus de discours comme l'indique le titre de l'œuvre.

2 Les noms de ces marionnettes signifient bien leur rôle dans le récit: le docteur Mabuse représente l'aspect négatif et dépressif d'un narrateur perturbé. Il provient à l'évidence du cinéma expressionniste allemand et plus précisément du film Docteur Mabuse le joueur (1922), de Fritz Lang, avec son docteur criminel qui veut dominer la ville entière de Berlin, voire l'humanité dans son ensemble. La deuxième marionnette, qui porte le nom de Robin Hood, est à relier au héros médiéval, aventurier défenseur des opprimés et des pauvres gens, sorte de Don Quichotte avec lequel le narrateur s'identifie. La troisième marionnette, la Wendy, est une référence à la Wendy Darling du roman Peter Pan et du film de Disney, à la fois ingénue, fuyante et mielleuse.

3 Dans La Nave de Baco Sánchez-Ostiz reprend cette conception de l'auteur ventriloque: «Me parece que escribir es arte de ventriloquia» (Sánchez-Ostiz, 2004: 14). 
Autrement dit, le narrateur traditionnellement unique, omniscient ou neutre, devient ici une voix multiple, derrière laquelle il se cache. Comme le précise Sánchez-Ostiz lui-même:

Ahora pienso que La flecha del miedo debería haberse escrito entera con las voces de esos tres muñecos. Era la manera de hablar de asuntos propios, algunos graves, sin esa crudeza, exhibicionista de las falsas confesiones. ${ }^{4}$

Nous passons ainsi du monologue de Perico, le crieur public de Las pirañas, à cette voix complexe de La flecha del miedo que manie ce ventriloque singulier. Dans cette vision terrible de la réalité provinciale, Sánchez-Ostiz a souvent recours à la caricature comme moyen d'expression, avec une déformation systématique de la réalité qui engendre une esthétique des «espejos cóncavos» comme disait le Max Estrella de Luces de Bohemia.

C’est ainsi que s'entrecroisent, dans le même texte, d'une part le monde réel, accompagné de sa violence meurtrière dans les rues, et social avec ses injustices, et de l'autre l'univers de l'imaginaire. Le narrateur, alter ego évident du romancier, revendique clairement le droit au rêve: "Soñar, vivir al margen, inventar, nunca pide vivir en otra parte, doctor, alérgico total... soy un náufrago, un Robinson » (Sánchez-Ostiz 2000: 216). Il proclame de façon catégorique: «La fantasía ése sí que es un paraíso perdido» (ibid.: 216) pour échapper à la flèche de la peur, de l'asile dans lequel on veut l'enfermer par la force:

Igual las cosas no son como yo las recuerdo, pero no puedo hacer otra cosa. Están ahí, en mi memoria, son mis ruidos [...]. Visto desde ahora todo aquello me parece como algo poco o nada vivido. Una de las muchas lagunas que hay en mi vida y que no podré rellenar jamás, a no ser que me lo invente todo, de arriba abajo, que es la mayor subversión que conozco, la rebelión radical: inventarse todo, el presente, el pasado, el futuro, contra todos y contra uno mismo, sobre todo contra uno mismo, y coger la bocina, asomarse por la borda y berrearle a la tempestad:

«Eh, los del barco!» «QQuéééé?»

«Iros a la mierda», porque ése es el barco de los muertos vivos, el verdadero buque fantasma, el que si te agarra vas avisado. (Sánchez-Ostiz 2000: 417-418)

Umbría, cadre spatial de l'histoire, est une ville fictive qui représente symboliquement Pampelune de la même façon que Vetusta incarnait Oviedo dans La Regenta de Clarín. En définitive, elle est l'image, une métaphore du Pays Basque et même de l'Espagne entière:

Y la ciudad, la mía, la que es de verdad mía, Umbría, a lo lejos esta vez, al otro lado del río, al otro lado de las vías del ferrocarril, al otro lado, en una verdadera tierra de nadie. Vista, entrevista, recordada en el papel, habitada por fantasmas, definitivamente. (Sánchez-Ostiz 2000: 9).

Cette ville qui n'est pas nommée dans les premiers romans comme Las pirañas, mais toujours présente cependant, trouve une plus grande force avec cette désignation imaginaire et symbolique. Comme Sánchez-Ostiz l’a avoué à Anna

4 Correspondance personnelle du 06/12/2011. 
Caballé: «Cuando pienso en Pamplona pienso en una ciudad casi invisible, la de la memoria, del pasado, la de la historia, una ciudad de papel [...]» (Caballé 1996: 19).

À ce monde virtuel s'oppose celui, dur et agressif, de la police anti-émeutes qui arrête le ventriloque, le frappe et le conduit devant la justice. Mais, au bout du compte, tout n'est que rêve, et il se réveille dans son lit:

Salí fuera y me dirigí al descampado en busca de la troupe de aquel Magic Circus que sólo parece tener existencia en mi memoria. Ya se habían ido, pero ido del todo. Sólo quedaba un resto de hoguera, desperdicios.(Sánchez-Ostiz $2000: 579$ )

Il a donc désormais choisi et trouvé son vrai chemin, il peut fermer le Théâtre de la Lune, est advenue l'heure du réel, c'est-à-dire de laisser la magie et de prendre des trains qui partent vraiment. Le récit se termine sur une vision plus prosaïque: «El tren con destino a Pamplona va a efectuar su salida... Telón y cuenta nueva» (Sánchez-Ostiz 2000: 591). Pampelune remplace Umbría.

\section{La métaphore de la ville}

Dans un roman qui semble profondément ancré dans la réalité humaine, sociale et historique de la Navarre et du Pays Basque, le choix d'un nom fictif prouve un refus de tout réalisme socio-historique, et même du simple "costumbrismo", représentation des mœurs, pour passer à un territoire qui se transforme en un mythe littéraire et dépasse largement le cadre provincial pour toucher à l'universel. D'où la vision grotesque qui ouvre le récit avec ces chiens qui aboient dans la nuit: "Perros que guardan casas cerradas, deshabitadas desde hace mucho tiempo» (Sánchez-Ostiz 2000: 9). Le narrateur est perdu dans ce monde fantasmagorique:

Y lo más intenso, lo más barbis, lo de un gran dolor le mordía como un lobo el corazón. Los oyes, al fondo, siluetas oscuras, voces desgarradas, barriendo las calles vacías, barriendo la cubierta de la nave de los locos, ancho camino de la muerte en vida, tan-le-al-com-pa-ñe-ra... y siguen y siguen y siempre acechan detrás de una esquina. Y lo cierto es que nadie sabe su historia, ni siquiera mis muñecos. (Sánchez-Ostiz 2000: 36)

Sánchez-Ostiz a confirmé, dans un Blog récent, le rôle que joue le brouillard dans son œuvre, donnant au passage la clé de sa relation étrange avec la ville:

Podría decir que me gusta la ciudad en la que vivo con niebla porque no la veo, pero eso es una malevolencia superflua; al revés, la veo mejor, como al hombre invisible de H. G. Wells, a quien sólo se le veía el rostro cuando fumaba. La niebla dulcifica los perfiles de las cosas, impone un silencio y una quietud raros: el engaño de que la realidad se amansa y el tiempo que muerde con ellas. No veo la ciudad, pero la imagino, entre realidad y sueño, entre lo vivido y lo imaginado, en ese dédalo de calles donde están las cosas y las personas que hemos perdido o nos han arrebatado: Biargieta, en su trazado voy viviendo. (Vivir de buena gana, blog, 12/01/2012) 
Le brouillard toxique sert à synthétiser sa singulière vision de la ville. Et une fois parcouru ce chemin rendu pénible par les circonstances contraires, le brouillard apparaît à nouveau pour visualiser les amis disparus: "Porque en Umbría los muertos de la cuadrilla quedan flotando a nuestro alrededor como nieblas espesas y se hacen del viento de las palabras en las grandes ocasiones » (SánchezOstiz 2000: 552).

Avec ce livre, le cycle entamé en 1992 avec Las Pirañas se ferme, de façon logique et cohérente, tandis que s'en ouvre un autre, annoncé sous le titre générique Las armas del tiempo qui doit théoriquement se poursuivre jusquà la guerre civile 5 .

Le titre El corazón de la niebla, premier tome de la nouvelle série, est très significatif et atteste la continuité entre les différents livres: ce Juan Miguel Arróniz, dont le narrateur essaie d'expliquer la mort mystérieuse, vivait dans une ferme dans la vallée de Humberri où il sétait réfugié, désabusé aussi bien de la politique, au début de l'étape socialiste, que de la vie, après la disparition de son épouse:

Así que Juan Miguel Arróniz, sin encomendarse ni a Dios ni al diablo, quemó sus naves y se fue a Humberri, y aquel mundo de luz líquida, sobre todo en otoño, y de nieblas y de lluvia tan fina que parece no caer, sino quedar suspendida en el aire, fue a partir de entonces el suyo. (Sánchez-Ostiz 2001: 79)

On peut affirmer que l'image du brouillard est centrale dans ce roman presque policier qui dévoile parfaitement les secrets de ce personnage énigmatique, à la dérive, «máscara de sarcasmo e ironía» (Sánchez-Ostiz 2001: 16), homme revenu de tout, «extraviado en la niebla» (ibid.: 185) et représente la difficulté de l'enquête lancée par le narrateur-avocat qui reconnaît lui-même à un certain moment que «La niebla, una vez más, le estaba jugando una mala pasada» (ibid. : 195).

Comme toujours chez Sánchez-Ostiz, se développe ici une satire impitoyable de l'hypocrisie, de la corruption et de la vanité dans le domaine de la vie politique, sociale ou littéraire. Le brouillard en vient à être un personnage comme les autres et provoque de façon symbolique la mort du philosophe: "Y la niebla iba a atrapar a Juan Miguel Arróniz. Le iba a rodear poco a poco, una estación detrás de otra, hasta ahogarlo en su seno" (Sánchez-Ostiz 2001: 117). Et il dépasse le simple élément climatologique caractéristique de la zone du Nord de l'Espagne pour prendre un sens métaphorique: «En la niebla pasan y pueden pasar cosas más extraordinarias» (Sánchez-Ostiz 2001:148). Le narrateur, une fois encore miroir de Sánchez-Ostiz, conclut avec cette affirmation qui résonne comme un véritable manifeste: "Creo que las cosas que de verdad nos perturban quedan flotando en el aire a nuestro alrededor de una manera rara,

5 En 2013, Sánchez-Ostiz a publié El escarmiento qui poursuit ce cycle consacré à la guerre civile, dont il annonce une suite sous le titre de El Botín. 
insidiosa, como si fueran una niebla espesa» (Sánchez-Ostiz 2001: 281). C'est donc la littérature qui se rapproche le plus de la vie, face à la loi du silence qui l'empêche de découvrir la vérité à propos de la mort de Arróniz, son ami:

Yo quería saber algo más de todo aquello y me tropecé con una muralla curiosa. Una muralla que unas veces era de piedra y otras de niebla, pero en los dos casos, impenetrable. (Sánchez-Ostiz 2001: 235).

Ce roman reconstitue, selon la technique narrative habituelle dans les textes de Sánchez-Ostiz, la rencontre entre deux vaincus, deux naufragés solitaires dont les vies se croisent et se trouvent au-delà du temps.

C'est de la même façon que le narrateur de En Bayona bajo los porches (Sánchez-Ostiz, 2002) se présente au lecteur comme un homme "naufragado en la melancolía» (ibid.: 12), qui écrit ce récit pour «no naufragar del todo» (ibid.: 13) comme son ami, Miguel Arróniz, objet de l'investigation du livre précédent. Refugié dans un moulin, il est devenu collectionneur et vit «en la niebla de sus días» (ibid.: 14). Il a la sensation de "vivir en un laberinto, en un jardín de caminos cruzados» (ibid.:14) et dans une «extraña tela de araña» (ibid.: 15), série de métaphores qui suggère son état d’âme.

En recomposant cet imbroglio, que constitue la vie de Tristan de Barraute, aristocrate bohème et extravagant du XIx ${ }^{e}$ siècle, en se fondant sur les archives, il sort de sa propre routine: il consulte des photographies et des lithographies de la troisième guerre carliste, une gravure achetée aux Marchés aux Puces de SaintOuen, qui reproduit le visage fascinant de l'aventurier, ou un livre relié avec la fameuse gravure que lui offre un ami de Pau, dans lequel on raconte les aventures turques et vénitiennes de Barraute. Il récupère des lithographies qui reproduisent ses combats en Russie et en Turquie; il découvre des photographies à Bayonne quartier général des carlistes, d’où le titre qui symbolise parfaitement les aléas de la recherche des traces de cet être singulier engagé dans «las armas del tiempo" (ibid.: 94), expression que l'on retrouve dans le titre générique du cycle qui a commencé avec El corazón de la niebla et se poursuit avec En Bayona bajo los porches, donnant à l'ensemble une cohérence thématique qui dépasse l'anecdote ${ }^{6}$.

Le Jeu de l'Oie est un autre élément commun aux deux livres: il réapparaît ici avec la même signification quand son amie antiquaire, Cuca del Río, lui offre un jeu semblable qu'il définit comme une "representación del recorrido vital» (ibid.: 104) avec ses ponts, son auberge, son puits, son labyrinthe, sa cellule et la mort que son ami Fermín Mugueta enrichit en y ajoutant un jeu de cartes de tarot et des idéogrammes du I-Ching.

Il s'agit en fait d'un roman sur le roman que fut la vie de Barraute: les références à des sources historiques précises en rapport avec des événements réels, depuis

6 La nave de Baco est le troisième tome de cette série. Dès un «Aviso para caminantes» qui ouvre le roman, celui-ci est revendiqué comme «una obra de ficción, una farsa, un guiñol burlesco.» (Sánchez-Ostiz 2004: 11) 
les guerres carlistes jusqu'aux épisodes dramatiques de Montejurra en 1976, ne doivent pas occulter le plaisir que l'auteur-narrateur éprouve à recréer un monde de figures extraordinaires, de chasseurs d'expériences exotiques parmi lesquels évolue Barraute et, par la même occasion, celui du lecteur qui peut et doit lire ce livre avec jouissance, comme un récit d'aventures, et le recevoir comme s'il était du domaine de l'imaginaire. Dans cette enquête, longue et tenace, les références à des documents iconographiques sont permanentes et le narrateur est à la fois bibliophile, historien et archiviste. Et nous restons sur une image qui domine, celle d'un authentique personnage de fiction, une espèce de Don Quichotte:

Su silueta, proyectada en la pantalla de papel de las páginas escritas o por escribir, invita a rellenar los silencios narrativos de su vida, mientras que la vida de Madrid [...] es parte de un presente que, a ratos, me resulta ininteligible, cuando no agobiante. (SánchezOstiz 2002: 485)

La reconstruction de la vie de Tristán de Barraute, «extraviado en su propio siglo» (ibid.: 17) ami des montreurs de marionnettes, devient pour le narrateur une manière de lutter contre la vie «cuando creía que había naufragado definitivamente en la rutina» (ibid.:18), vie présente définie justement par l'image de la brume. La consultation de sa biographie écrite par le grand-père de son ami Bordenave le conduit à des réflexions sur les rapports complexes entre la biographie et l'Histoire. Il plaide en faveur d'un mélange entre les deux, en revendiquant la présence nécessaire de l'imaginaire, ce qui donne naissance à un genre nouveau qu'il appelle "una biografía novelesca» (ibid.: 309). Il compare lécriture et le discours littéraire à un jeu de l'oie: narrer la vie de Tristán de Barraute lui donne l'illusion du voyage et le métamorphose. Cela lui a transmis des envies de sortir du chemin de la raison et de se laisser mener "por el azar del juego» (ibid.: 482), à l'instar de ce qui se passe dans le jeu de l'oie: «Barraute es un acicate para echarse al camino en pos de sus huellas, una invitación al viaje, aunque ese viaje sea literario» (Sánchez-Ostiz 2002: 485). Il élabore, de cette façon, une conception personnelle du roman qui doit faire appel à l'imagination, aussi bien pour évoquer la vie d'une personne réelle que pour raconter un voyage. Il dit: «La invención suele estar más cerca de la realidad de lo que parece» (ibid. : 484). Au cours de ses enquêtes, il est devenu l'ami d'un certain Martín Aguirre, qui lui fait part d'un projet de recherche d'un trésor perdu dans le naufrage d'un navire près de l'île de Juan Fernández que l'on considère souvent comme la probable île où fit naufrage et vécut Robinson. Curieusement les deux livres suivants de Sánchez-Ostiz vont raconter une expédition de lécrivain au Chili et jusqu'à cette île qui le fascine, à la poursuite du mythe de Robinson Crusoé. 


\section{Le Mythe de Robinson}

La isla de Juan Fernández (2005), La calavera de Robinson (2006) et Cuaderno boliviano $(2008)^{7}$ sont des textes écrits à la suite de séjours de l'auteur dans le continent américain. Ils sont significatifs de l’activité polyfacétique de SánchezOstiz qui, à partir de la même expérience, propose des œuvres qui appartiennent à des genres différents ${ }^{8}$.

La première est le traditionnel cahier de route d'un écrivain qui découvre la réalité géographique et humaine de cette île, nouvelle pour lui, fascinante par son lien mythique avec le personnage de Robinson Crusoé. La deuxième, publiée seulement deux années plus tard, est un authentique roman, au-delà d'une possible répétition du même sujet, c'est-à-dire une fictionnalisation dépisodes réellement vécus, tandis que la troisième est le classique journal de voyage vers une île sur laquelle il pose un regard honnête et ouvert. Bien entendu, le langage métaphorique sera plus présent dans le roman que dans les cahiers.

Quand Daniel Defoe publia Robinson Crusoé en 1719, il est alors un journaliste pamphlétaire anglais, d’origine flamande, dont l'entreprise maritime a fait faillite en 1692, et qui se lance dans une carrière littéraire à la suite de cet échec économique. Pour créer son personnage qui va devenir un des plus grands mythes de la littérature universelle, il s'inspire d'un fait réel, l'infortune d'un marin écossais, Alexander Selkirk, que son capitaine a abandonné, parce qu'il nobéissait pas, sur le rivage de cette île du Pacifique, face au Chili, où il ne resta que quatre ans et quatre mois, entre 1704 et $1709^{9}$.

L'idée géniale de Defoe fut de forger une histoire sur la base de cet épisode réel, assez courant à l'époque. Dans sa version, Alexander Selkirk se transforma en Robinson Crusoé, son séjour dura 28 ans et deux mois, et se fonda aussi un duo avec un être natif de cette île, Vendredi. Le texte prend la forme d'un journal de bord rédigé par le marin pendant ce long séjour. Defoe crée donc une fiction à partir de cette anecdote vraie mais en lui donnant un fort effet de réel. Il est symptomatique que Sánchez-Ostiz consacre deux livres à ce héros extraordinaire qu'il récupère pour exprimer ses propres interrogations et obsessions.

Defoe était un vaincu solitaire quand il a écrit son livre et l'histoire qu'il reprend devient un hymne au courage, à la ténacité et à la capacité de l'homme à s'adapter et à surmonter les obstacles. Sánchez-Ostiz entreprend dans $L a$ isla de Juan Fernández, une fois encore, une enquête policière, tel un détective, pour

7 Cuaderno boliviano appartient au genre des chroniques de voyages, à mi-chemin entre le journal intime, et le reportage politique, fruit de séjours répétés de l'auteur en Bolivie depuis 2003.

8 On peut observer chez Sánchez-Ostiz une véritable frénésie d'écriture: dans le cas de l'île de Juan Fernández il en vient à publier, à des dates très rapprochées, des ouvrages consacrés au même sujet. Par exemple, La isla de Juan Fernández en 2005 et La calavera de Robinson en 2006.

9 On sait que ce livre inspira plus tard à Jules Verne son Île mystérieuse (1874) et à Robert Louis Stevenson son Île au trésor (1883). 
reconstituer le destin de cet aventurier afin de tirer de son destin une leçon morale. Il s'intéresse donc à la recherche de l'espace authentique où le marin vécut isolé, grâce à une expérience directe sur le terrain, à partir de ses excursions dans l'île. Il cherche à comprendre qui fut exactement cet Alexander Selkirk, en lisant tous les récits qui racontent les circonstances d'un naufrage et il interroge une série d'individus étranges qui habitent aujourd'hui dans cette île.

Ici l'aspect documentaire de l'enquête est important comme le suggèrent le titre: La isla de Juan Fernández et le sous-titre: Viaje a la isla de Robinson Crusoe, la reproduction d'une photographie de l'île sur la couverture de l'édition, les cartes présentées au début, une note bibliographique complète à la fin. Dans La calavera de Robinson, il a recours à une image, celle du mystère de cette tête de mort.

Nous avons donc, dans les deux cas, une alternance entre d'une part le traditionnel journal de voyage depuis Santiago et le port de Valparaíso jusquà l'île de Robinson Crusoé, dans lequel Sánchez-Ostiz raconte à la première personne les conditions compliquées du trajet, l'installation dans les auberges de l'île, les promenades à la découverte de la nature sauvage, aride et hostile, les rencontres et conversations avec des personnages bizarres, et de l'autre des réflexions sur la vie, le temps qui passe, la solitude. Il analyse surtout ce que représente cette île qu'il associe à ses rêves d'enfant: pour lui, elle est « una imaginaria puerta de la fuga. Decir Juan Fernández es decir lejanía» (Sánchez-Ostiz 2005: 14).

Ce contraste entre le réel et le rêve, entre ce qu'il observe, une terre inhospitalière, avec des loups marins impressionnants, et la légende paradisiaque, est la base du mythe qui fascine Sánchez-Ostiz, c'est un appel à l'imaginaire. L'île est une image qu'il porte en lui depuis les lectures juvéniles et qui le poursuit encore, dans l'espace mystérieux de songes, c'est un lieu fantasmagorique, théâtral, celui de tous les possibles. Il se place donc dans le courant de ses modèles littéraires: Stevenson, Mac-Orlan, Melville, Conrad ou dans celui de ces marins intrépides, ces pirates aventuriers des temps anciens, ces archéologues, chercheurs d'or, restaurateurs, collectionneurs, peintres extravagants du temps présent:

Desde ese momento, y durante años, los barcos atestados de soñadores, aventureros, hampones, jugadores de ventaja, proscritos, miserables, visionarios que hacían la travesía a América pasando forzosamente por el cabo de Hornos, no hicieron sino pasar y repasar por Juan Fernández. (Sánchez-Ostiz 2005: 308)

Il en tire la leçon qu'en définitive «se impone la realidad de que es mejor escribir sobre lugares imaginarios» (ibid.: 332).

À la fin de La isla de Juan Fernández, il parle des témoignages de quelquesuns de ces aventuriers comme l'Italien Jorge Di Giorgio en 1950, le navigateur R. E. Dan en 1834 ou l'écrivain américain Ross Browne, en 1848. Ce dernier fait même allusion au trafic de la tête de mort de Robinson qui devient le moteur essentiel du roman suivant, comme le suggère explicitement le titre: La calavera de Robinson. Dans un étrange prologue, appelé Paraphrase, il est dit au lecteur: 
Antes de darle la voz al Narrador, no está de más parafrasear, a modo de homenaje, a Daniel Defoe, sin cuyo inestimable concurso esta novela no habría sido posible [...] (Sánchez-Ostiz $2007:$ 7)

Le narrateur se présente comme un collectionneur fou d’objets rares, ébloui par les secrets de l'île de Juan Fernández qu'il découvre grâce à l'héritage d'un aventurier du nom de Telmo Camacho qui lui lègue sa Crusoniana, une collection d'objets insolites. Parmi eux trône une tête de mort qu'il prétend être celle de Robinson, autrement dit celle d'un personnage inventé. Il décide alors de partir pour l'île à la recherche de la vérité du trésor caché que Camacho aurait trouvé dans la mythique île.

Certes, si nous avons aussi un journal de voyage, assez semblable à celui que nous avons déjà eu dans La isla de Juan Fernández, l'enjeu de ce livre, son ambition, sont différents, ils sont plus complexes et profonds. En créant cette histoire, Sánchez-Ostiz donne plein pouvoir à l'imagination et affirme sa conception particulière du roman: «Un novelista levanta en su imaginación mundos imaginarios que sólo se hacen visibles en sus palabras» (Sánchez-Ostiz 2005: 136).

La tête de mort de Robinson symbolise, dans son urne, cette recherche qu'entreprend le narrateur, elle est l'image du voyage dans un monde imaginaire, l'icône des batailles menées par Camacho tout au long de ses aventures dans l'île, la représentation de sa personnalité de «embaucador de categoría, un fantasioso, un soñador, alguien que se había dado maña para cubrir la mugre de su existencia con unos fantasmas que le permitían, sobre todo, admitirse» (ibid.: 43). Le but est donc de dévoiler les secrets cachés de ces vies aventurières et de trouver un sens à l'existence perturbée du narrateur dominé par «ese hastío de las cosas, esa fatiga de uno mismo» (ibid.: 385). Il se sent le frère de ce Camacho qui mène au Chili une vie «fantástica» (ibid.: 27). Et qui lui ouvre les portes de la faculté d'invention de Robinson.

Les objets qu'il a collectionnés (cartes, coupes pages, vues de la Baie de Cumberland, gravures, longues-vues, pièces de monnaie, bureaux) l'introduisent dans le monde fascinant de ces marins escrocs, mythomanes, flambeurs, anthropologues, botanistes, antiquaires, peintres, tous ces Juan Uribe, John Ross Browne, William Pierce, Auguste Duval, Salvador Reyes Figueroa, Marcos Moltedo o Mauricio Gamboa qui tentent désespérément de sortir de leur routine, leçon permanente des textes de Sánchez-Ostiz.

Finalement, il semble que l'histoire de la tête de mort de Robinson est «sólo una fantasía literaria" (ibid.: 114), plus sujet d'histoires ou attraction de foires que réalité historique. Une formule résume tout cela et définit parfaitement la conception du roman de Sánchez-Ostiz: « ¿Importa cómo fueron las cosas? ¿O importa cómo las imaginamos?» (ibid.: 381 ).

Au retour, chez lui, le narrateur tombe amoureux d'une femme, Sonia Ferrer, spécialiste en momies et rites funéraires et tous deux se retrouvent dans leurs "caminos bifurcados» (ibid.: 483). Nous ne sommes plus dans un journal de 
voyage mais dans un roman, nous constatons que tout est fiction et que cette fiction explique mieux la réalité: «[...] la invención, sea o no literaria, puede acercarse tanto a la realidad, que acaba por sustituir a ésta» (ibid.: 491)

Cornejas de Bucarest est l'exemple parfait du malentendu entre Sánchez-Ostiz et la critique littéraire en Espagne qui s'obstine à recevoir ce genre de livres comme un journal personnel, contrairement à ce quaffirme clairement l'auteur dans un Avis préliminaire:

Nunca está de más aclarar que esta es una obra literaria en que se narra una acción fingida cuyo fin es causar placer estético [...] todos los personajes y situaciones son por completo imaginarios e improbables, tanto el narrador y, por supuesto, el autor. (Sánchez-Ostiz 2010: 8)

Le titre définitif Las cornejas de Bucarest plutôt que celui qui était initialement prévu, Pícaros en Bucarest, montre bien que l'on passe d'une référence plutôt réaliste, et bien codée, avec la picaresque, à quelque chose de l'ordre de la métaphore. Ces corneilles observées dès le jour de son arrivée sont, pour le narrateur la représentation symbolique d'une ville fantasmagorique, froide, plongée dans le brouillard et couverte de neige, avant de disparaître elle aussi.

Certes, on retrouve le même point de départ: des carnets de voyage qu'un écrivain rédige à l'occasion d'un long séjour professionnel à Bucarest. Nous le suivons au fur et à mesure de son installation dans ses résidences, sa découverte des rues et de lieux nocturnes de la capitale de la Roumanie.

On voit resurgir les thèmes habituels chez Sánchez-Ostiz: la prise de contact avec un univers inconnu, hostile, dompté peu à peu au prix d'un vagabondage quotidien dans les rues, la recherche de lieux authentiques, très souvent nocturnes, et marginaux, des librairies, des cafés, des bouquinistes, et la découverte et pratique d'un monde de gitans, de prostituées, d'escrocs, de mendiants, bien loin des clichés pittoresques des dépliants touristiques.

Le point de vue est, encore une fois, celui d'un écrivain fatigué, sceptique, vaincu, en proie au doute, malade et fragile, cherchant dans ces expériences des raisons de résurrection. La représentation du monde institutionnel de la culture, l'Institut Cervantès en particulier, est iconoclaste, terriblement critique avec la vision d'un microcosme de vaniteux, de corrompus et d'inutiles, perçu comme une farce burlesque. Il dénonce les «canalladas habituales del Centro de las Letras Españolas y del turismo académico y ministerial» (Sánchez-Ostiz 2010: 157).

Face au roman historique en vogue, Sánchez-Ostiz se situe dans le courant picaresque. «Indudablemente por su desgarro, por su nihilismo, por su fatalismo, por la rebeldía última del contarlo, en un mundo, aquel, en el que todo es mordaza» (ibid.: 160). Il plaide pour «El desarraigo, la necesidad de vagabundeo, de merodeo [...] la atracción de lo prohibido» (ibid.: 161) et conclut par ce qui peut apparaître comme un Manifeste Littéraire: «[...] reventemos el ceremonial del lenguaje, tan hermoso como hueco, las pijadas de los estetas» (ibid.: 161). 
Cornejas de Bucarest n'est donc pas un roman historique, non plus qu'une simple fiction ou une autobiographie. Peut-être se rapproche-t-on, avec ce livre, de l'autofiction car c'est aussi un texte très imaginatif. D'où cette série d'aventures invraisemblables que vit le narrateur: une relation amoureuse avec une cavalière de cirque, des orgies nocturnes dans des cabarets où règne la drogue, mélangée à des souvenirs de son passé d'avocat dans l'Espagne de 1982. On y croise des personnages romanesques comme un professeur de roumain, un poète chilien, une journaliste culturelle, un légionnaire. On se retrouve en pleine fantaisie, loin de la vie de l'auteur; une autre vie, imaginaire, un puzzle très compliqué se créent. La narration de la vie de cet écrivain espagnol à Bucarest, sous la forme d'un journal de voyage, sert de base à la création d'un monde dominé par l'invention et l'intensité d'un discours baroque.

\section{Conclusion}

En définitive, le genre défendu et illustré par Sánchez-Ostiz n'est vraiment pas le réalisme descriptif et social mais plutôt la poésie avec ce recours systématique aux techniques d'intensification qui fait du texte non une représentation de la réalité mais une visualisation déformatrice de celle-ci et même parfois une théâtralisation. C'est ainsi que l'on trouve de nombreuses images, des procédés d'emphases avec des énumérations, des comparaisons et des accumulations et répétitions qui nous éloignent du style neutre du récit historique.

Lauteur ne prétend pas seulement dénoncer la société contemporaine mais en donner une métaphore, par l'utilisation de tous les procédés de la rhétorique littéraire. La clé des romans de Sánchez-Ostiz n’est pas la remémoration, historique ou journalistique, mais le "esperpento", c'est-à-dire la déformation grotesque et dégradante de la société roumaine ou espagnole dans la tradition du Valle-Inclán de Luces de Bohemia. Avec cette représentation singulière, il ne s’agit pas de donner une image déformée de la réalité mais de fournir au lecteur l'image fidèle d'une réalité par elle-même difforme.

\section{Bibliographie}

Canallé, A, 1996, Boletín de la Unidad de Estudios Biográficos, Entrevista a Miguel Sánchez-Ostiz, Barcelona, PPU, número 1, Enero de 1996.

Sánchez-Ostiz, M., 2000, La flecha del miedo, Barcelona, Anagrama. Sánchez-Ostiz, M., 2001, El corazón de la niebla, Barcelona, Seix Barral. Sánchez-Ostiz, M., 2002, En Bayona, bajo los porches, Barcelona, Seix Barral. Sánchez-Ostiz, M., 2004, La nave de Baco, Madrid, Espasa Calpe. Sánchez-Ostiz, M., 2005, La isla de Juan Fernández, Barcelona, Ediciones B. Sánchez-Ostiz, M., 2007, La calavera de Robinson, Irún, Alberdania. Sánchez-Ostiz, M., 2010, Cornejas de Bucarest, Pamplona, Pamiela. Sánchez-Ostiz, M., 2011, Zarabanda, Pamplona, Pamiela. Sánchez-Ostiz, M., 2013, El Escarmiento, Pamplona, Pamiela. 